\title{
SELF-ORGANIZATION OF ATOMIC SAMPLES IN RESONATORS AND COLLECTIVE LIGHT FORCES
}

\author{
ADAM T. BLACK, HILTON W. CHAN, VLADAN VULETIĆ \\ Department of Physics, Stanford University, Stanford, \\ CA 94305-4060, USA \\ Current address: Center for Ultracold Atoms, Massachusetts Institute of Technology, \\ Building 26, 77 Massachusetts Avenue, Cambridge, \\ MA 02139-4309, USA \\ E-mail:vuletic@mit.edu
}

\begin{abstract}
We report on a novel type of collective friction force acting on the center-of-mass motion of a self-organizing atomic sample inside a resonator. The force substantially exceeds the sum of the light forces acting on isolated atoms. We also observe spontaneous symmetry breaking between two equivalent, but spatially offset self-organization patterns in the standing wave resonator, as predicted by Domokos and Ritsch. We identify and experimentally verify the dependencies of the threshold for the self-organization process on incident-light and atomic sample properties.
\end{abstract}

\section{Introduction: Single-atom versus collective light forces}

The well-known light-induced forces on atoms, such as the conservative dipole force, or the dissipative Doppler and polarization gradient forces ${ }^{1}$, arise from the interaction of the incident light with single atoms. Collective parameters characterizing the sample, such as the number of atoms, their spatial or momentum distribution etc., are irrelevant to the problem, and the forces on the sample, or parts of it, can be calculated as the sum of the corresponding single-atom forces.

The justification for treating the atoms in the gas as independent scatterers of radiation is the random distribution of atomic positions. In this case the fields emitted by most of the $N$ atoms interfere destructively, but random fluctuations of order $\sqrt{N}$ in the atomic spatial distribution produce an emitted field $\sqrt{N}$ times as large as the field emitted by a single atom. This results in a scattered power that scales as the number $N$ of atoms in the gas, which justifies the independent-emitter picture $^{2}$.

There are, however, well-known many-atom systems where the fields emitted by different atoms interfere constructively, and where consequently the emitted power is proportional to $N^{2}$, rather than to the atom number $N$. Such collective enhancement can occur in systems with inversion, where the phase of the emitted light is free to adjust, such as in Dicke superradiance ${ }^{3}$, or in the form of Bragg scattering from spatially ordered systems. Given that in laser cooling kinetic energy is removed from the sample in form of a frequency difference between the emitted and the incident light, a larger collective light emission rate opens the possibility for collectively enhanced cooling forces ${ }^{4}$. In contrast to conventional optical cooling methods, such a mechanism would become more efficient as the size or density of the sample is increased.

Here we report on a system where collective emission by a self-organizing sample into a resonator leads to a friction force acting on the sample's center-of-mass mo- 

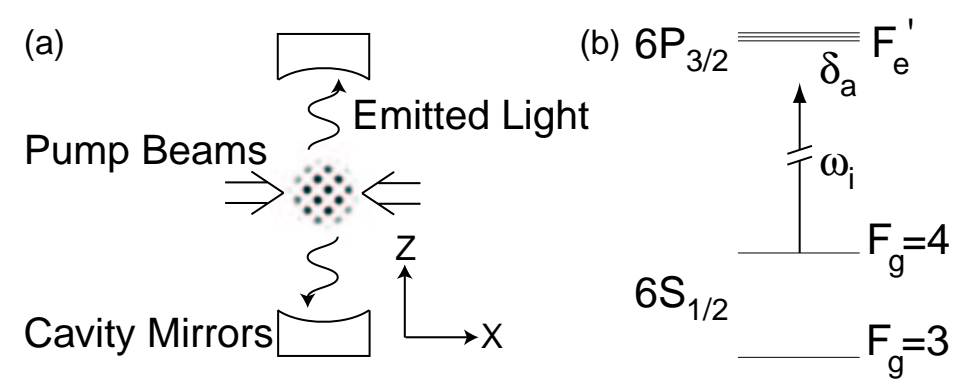

Figure 1: (a) Experimental setup. A sample of cold atoms falling along the axis of a near-confocal resonator is illuminated by a pair of horizontal, counterpropagating pump beams linearly polarized along the $y$ direction. (b) Cs $\mathrm{D}_{2}$ transition, with hyperfine structure and pump light frequency indicated. The light is far detuned compared to the excited-state hyperfine structure, resulting in an open two-level system on the hyperfine $F_{g}=4 \rightarrow F_{e}$ transition.

tion, the magnitude of which far exceeds the sum of the single atom forces obtained under the same conditions.

\section{Self-organization and spontaneous symmetry breaking}

The experimental system in which we observe collectively enhanced emission is a cloud of $N \approx 10^{7} \mathrm{Cs}$ atoms, loaded in a MOT on the vertically oriented axis of a standing-wave optical resonator. The near-confocal resonator is $7.5 \mathrm{~cm}$ long and has finesse $F=1000$. We extinguish the MOT and illuminate the freely falling atoms with a horizontal standing wave pump beam, while collecting the light emitted from the cavity on a photodiode. The pump light is red detuned by an amount $\delta_{a} / 2 \pi$ between $-1.5 \mathrm{GHz}$ and $-6 \mathrm{GHz}$ relative to the atomic $6 S_{1 / 2}, F_{g}=4 \rightarrow 6 P_{3 / 2}, F_{e}=5$ transition, large compared to the excited-state hyperfine structure. The detuning between the pump light and the $T E M_{00}$ cavity mode is labelled $\Delta_{c}$. The detuning range where transverse modes of the near-confocal cavity have appreciable coupling to the atomic sample is given by $-200 \mathrm{MHz} \leq \Delta_{c} / 2 \pi \leq 0$.

For low pump intensities, ordinary cavity enhancement ${ }^{5,6,7}$ of Rayleigh scattering is observed. Above a threshold pump intensity, however, we observe greatly enhanced emission from the atoms into the cavity ${ }^{8}$, with a ratio of scattering rate into the cavity $\Gamma_{c}$ to scattering rate into free space $\Gamma_{f s}$ as large as $2 \eta_{c}=\Gamma_{c} / \Gamma_{f s}=200$. This scattering ratio contrasts with a maximum $2 \eta_{s}=0.1$ for scattering into the cavity without collective enhancement.

We interpret the enhanced emission as Bragg diffraction from a spatially ordered atomic density distribution ${ }^{9,10}$. The atoms localize in the optical dipole potential formed by interference between the emitted intracavity light and the pump light 10. Localization in such a lattice automatically satisfies the Bragg condition for scattering from one lattice beam into the other. This emission process therefore runs away once initiated: the stronger the emission into the cavity, the better the localization of the atoms in the density grating, which leads to still stronger Bragg diffraction. The emission into the cavity is ultimately limited by the reverse process of Bragg diffraction out of the resonator, which becomes dominant once the 
(a)

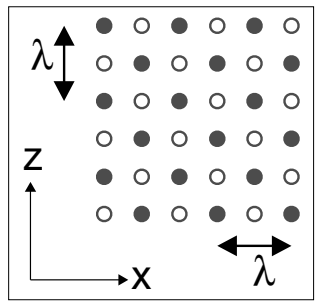

(b)

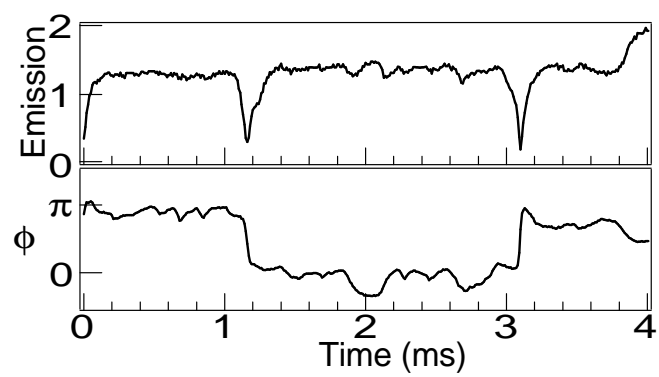

Figure 2: (a) The two possible lattice configurations producing relative phase shift $\Delta \phi=\pi$ in the emitted light. (b) Time trace of intracavity intensity, in arbitrary units, during illumination of the atoms by both a pump beam and MOT light. The parameters are atom number $N=8.2 \times 10^{6}$, light-atom detuning $\delta_{a} / 2 \pi=-1.59 \mathrm{GHz}$, light-cavity detuning $\Delta_{c} / 2 \pi=-20 \mathrm{MHz}$, and pump intensity (in terms of saturation intensity) $I / I_{s}=440$. (c) Time trace of the relative phase $\phi$ of the intracavity light, for the same experimental conditions.

intracavity intensity equals the pump intensity. We refer to this spatial ordering of the atoms as 'self-organization' because scattering from the atomic sample itself creates the intracavity field responsible for localizing the atoms in a two-dimensional grating.

As Domokos and Ritsch have pointed out ${ }^{10}$, two possible grating arrangements exist that lead to Bragg scattering into the cavity. The lattice sites form at the lines of intersection of the antinode planes of the pump light with those of the intracavity light. If there is constructive interference at one such intersection, the resulting 2D lattice has an antinode along that line, that constitutes a potential minimum for our red-detuned light. At the adjacent intersection of antinodes, one-half wavelength away in either the $x$ or $z$ direction, the two fields interfere destructively and there is no lattice site. The lattice therefore forms a chessboard pattern in the plane subtended by the incident light and the cavity (Fig. 2a). If one imagines that the time phase of the intracavity light now changes by $\pi$, this chessboard pattern inverts, so that field nodes become antinodes and vice versa. The new $2 \mathrm{D}$ lattice is equivalent to the original one, with all lattice sites translated in space by a halfwavelength.

The intracavity and pump standing waves exhibit symmetry under translations by $\lambda / 2$ in the $z$ and $x$ directions, respectively, in the region of interest. The selection of one or the other of the two grating configurations by the atoms therefore happens randomly. This spontaneous symmetry breaking can be explained by fluctuations in the initial spatial distribution of atoms, where one or the other of the lattice configurations has a stochastically larger population at the beginning of the emission.

The relationship between the time phase of the intracavity light and the location of the atomic density grating provides a means of probing the process of atomic selforganization ${ }^{11}$. In particular, changes to the lattice configuration are manifest in the time phase of the emitted light relative to the pump light. As a test of the model for self-organization, we measure this time phase by a heterodyne technique, both for free atoms and for atoms trapped in a MOT. For the untrapped atoms, the phase 
(a)

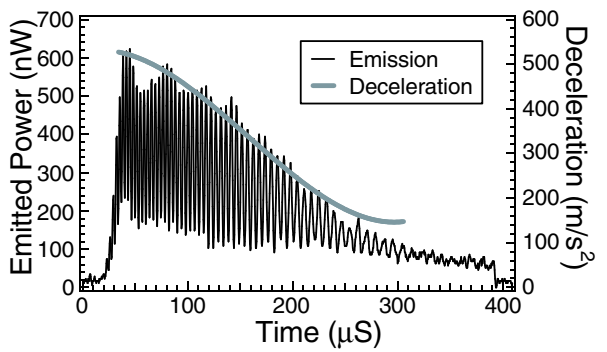

(b)

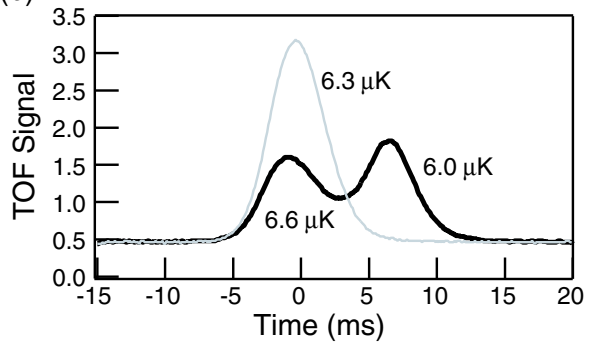

Figure 3: (a) The emitted power (thin line) during the illumination of a falling atomic cloud. The beat frequency is used to calculate the deceleration (thick line). Here the pump $I / I_{s}=420$, $\delta_{a} / 2 \pi=-1.580 \mathrm{GHz}$, and $\Delta_{c} / 2 \pi=-10 \mathrm{MHz}$. The atom number is $N=2.6 \times 10^{7}$. (b) The time-of-flight signal, in arbitrary units, of atomic clouds without (gray) and with (black) exposure to the pump beam. The initial MOT temperature, and the final temperatures of the delayed cloud and undelayed remnant are indicated.

of the intracavity light remains nearly constant, as expected for Bragg scattering from a stationary grating. If, however, a randomizing force is applied to the atoms by applying the MOT light, periods of nearly constant phase are interrupted by sharp phase jumps of approximately $\pi$ (Fig. 2c). These phase jumps correspond to spontaneous rearrangement of the grating, as we verify by observing that the Bragg scattering is strongly suppressed for the brief duration of the phase jumps (Fig. 2b).

\section{Collective cooling of the sample's center-of-mass motion}

The above measurements of phase jumps of the Bragg scattered light were performed with a stationary atom cloud. When the cloud is moving, as can be achieved by dropping the cloud for typically $20 \mathrm{~ms}$ before the pump is applied, we observe a strong modulation of the light emitted into the cavity at a frequency that equals twice the Doppler effect of the falling cloud (see Fig. 3a). The modulation can be understood in terms of the interference of the light emitted upward (with Doppler effect $-k v$, where $v$ is the downward velocity of the cloud), and the light emitted downward, with Doppler effect $+k v$. Alternatively, the modulation can be viewed as arising from the modulated emission into the cavity, as the self-organized matter grating falls past the cavity nodes and antinodes.

The decreasing modulation frequency in Fig. 3a indicates that the atomic sample is slowed during the collective emission. In fact, the slowing is also evident in time-of-flight (TOF) traces recorded for the falling cloud, where a substantial portion of the cloud, typically a third, is delayed in the arrival to the TOF sheet, indicating a reduction in velocity (Fig. 3b). The changing modulation frequency indicates decelerations of $300 \mathrm{~m} / \mathrm{s}^{2}$ for initial sample velocities of $15 \mathrm{~cm} / \mathrm{s}$, consistent with the decelerations obtained from the TOF measurements.

Preliminary data for faster clouds obtained by accelerating the atoms with a moving molasses ${ }^{12}$ to $1 \mathrm{~m} / \mathrm{s}$ indicate that at larger velocities even larger accelerations can be achieved, with observed values up to $5000 \mathrm{~m} / \mathrm{s}^{2}$. Note that the observed accelerations correspond to small fractional differences between the blue 
and red Doppler sidebands of only $1 \%$ of the emitted photons. If the sample can be made to emit on the blue Doppler sideband only, which should be possible for a velocity where the Doppler effect exceeds the cavity linewidth, and consequently only one sideband is amplified inside the cavity, even much larger decelerations are expected. Since the collective emission rate per atom can far exceed the Einstein A coefficient of the excited state while the saturation of the atomic transition remains small, the available collective cooling force for the center-of-mass motion should significantly exceed the maximum radiative (Doppler) force. Already we have observed forces whose magnitude exceeds the maximum conventional Doppler force at the given atomic velocity.

Given that the atoms self-organize into a density grating and Bragg scatter light cooperatively into the resonator, as observed in our experiments ${ }^{8,11}$, and confirmed and explained by the analysis and numerical simulations by Domokos and Ritsch ${ }^{10}$, we have developed a simple analytical model that can explain the cooling force on the center-of-mass motion that accompanies the Bragg scattering ${ }^{13}$. Viewed in the frequency domain, the cooling force stems from an asymmetry between the blue and red Doppler sidebands that is produced by simultaneous amplitude and phase modulation of the intracavity light by the falling grating, an effect similar to single-sideband modulation. This mechanism does not require a difference in cavity enhancement resulting from a cavity slope, as does cavity Doppler cooling ${ }^{14,15,16,17}$, but for red light-atom detuning occurs even if the cavity has the same response at the two sideband frequencies. The model shows quantitative agreement with the observed properties of the cooling force ${ }^{13}$.

\section{Collective force magnitude and threshold for self-organization}

If the observed force is to be used, e.g., for the slowing of new species at large detuning, the experimentally critical parameter is not the force magnitude, that can be very large due to the stimulated nature of the emission process, but the necessary intensity to reach threshold at the given particle polarizability, sample density and temperature, and light detuning.

To identify the origin of the optical gain and self-organization process, we have measured small-signal gain spectra using a weak auxiliary beam coupled through the cavity while pumping the system below threshold with the strong horizontal pump beams. For samples falling at velocity $v$ we observe two antisymmetric Gaussian profiles spaced by $2 k v$ (Fig. 4), characteristic of recoil-induced gain due to an inversion between momentum classes for a sample at finite temperature ${ }^{9,18}$. Assuming that recoil-induced resonances (RIRs) are indeed the dominant optical gain mechanism below threshold, we can derive the threshold condition for the system from the requirement that the RIR gain ${ }^{9,18}$ equal the cavity loss,

$$
\Gamma_{t h}=C \frac{k T}{\hbar \eta_{s} N_{e f f}},
$$

where $C$ is a coefficient of order unity, and for RIR gain is given by $C=C_{R I R}=$ $(e /(32 \pi))^{1 / 2}$. Here $\Gamma_{t h}$ is the single-particle Rayleigh scattering rate at threshold, $\eta_{s}$ is the probability for a single atom to emit a photon into the cavity (single-atom 

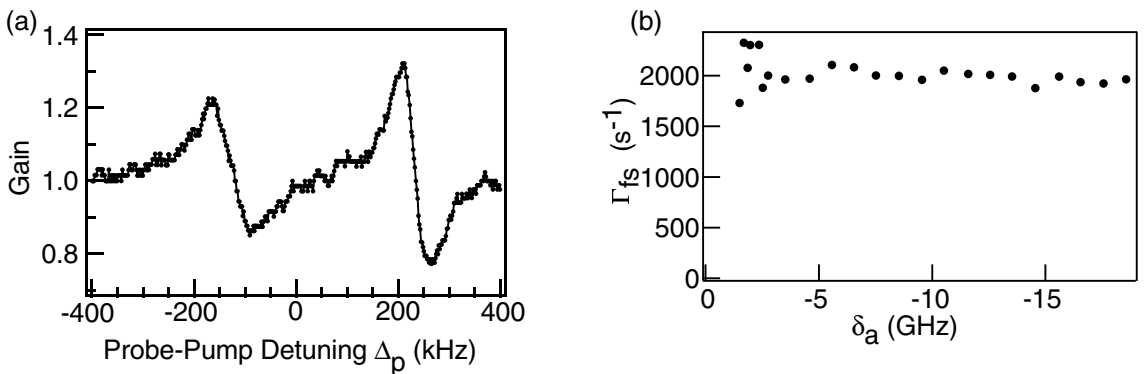

Figure 4: (a) Small-signal probe gain spectrum for a falling atomic cloud with velocity $v=15 \mathrm{~cm} / \mathrm{s}$, $N=2.5 \times 10^{7}, \delta_{a} / 2 \pi=-1.7 \mathrm{GHz}, \Delta_{c}=0$, pumped below threshold at $I / I_{s}=180$. (b) Threshold free-space scattering rate versus light-atom detuning. Atom number is $N=2 \times 10^{7}$, temperature is $T=5.0 \mu \mathrm{K}$.

cooperativity parameter), $T$ is the temperature of the sample, and $N_{\text {eff }}$ is the effective number of atoms coupled to the cavity mode (effective number of atoms at the cavity center producing the same response as the spatially extended atomic sample). If we treat the problem from a two-level superradiance viewpoint, where two momentum classes spaced by the photon recoil $\hbar k$ represent the two levels, we expect for the threshold the same formula with a slightly modified coefficient $C=C_{s r}=(e /(32 \pi))^{1 / 2} \ln N_{e f f}{ }^{19}$. The weak additional logarithmical dependence on atom number reflects the fact that the exponentially growing superradiant pulse into one electromagnetic mode needs to build up before the system decoheres by emitting into other spatial modes. Both models predict that the dependence of threshold on light-atom detuning and light intensity is only through the free-space Rayleigh scattering rate $\Gamma_{t h}$, and that $\Gamma_{t h}$ is proportional to the temperature $T$ of the gas, inversely proportional to the single-atom scattering probability into the cavity mode $\eta_{s}$, and predominantly inversely proportional to the atom number $N_{\text {eff }}$.

To verify the threshold dependencies derived from these simple models, we plot in Fig. 4b the measured single-atom Rayleigh scattering rate at threshold versus light-atom detuning for all other parameters, such as atom temperature, density or number, held fixed. The figure shows clearly that the detuning dependence enters only through the single-atom Rayleigh scattering rate.

To vary the atom number without affecting other parameters such as atom temperature or cloud size, we change the repumper intensity during the last phase of the MOT. This changes the atom number in the upper $F_{g}=4$ hyperfine manifold, that interacts strongly with the applied light red-detuned by typically $2 \mathrm{GHz}$ relative to the $F_{g}=4 \rightarrow F_{e}$ transitions. Fig. 5a shows that the threshold scattering rate is proportional to the atom number, in agreement with the prediction from Eq. 1. The additional logarithmic dependence on atom number of the superradiance model is too small to be observed within our accessible range of atom number variation. However, we do believe that the superradiant model that includes the pulse growth, rather than only the small signal gain, is closer to a complete description of the actual situation, which would involve a cascade of momentum states for each atom.

Finally, we measure the variation of threshold scattering rate with atom tem- 

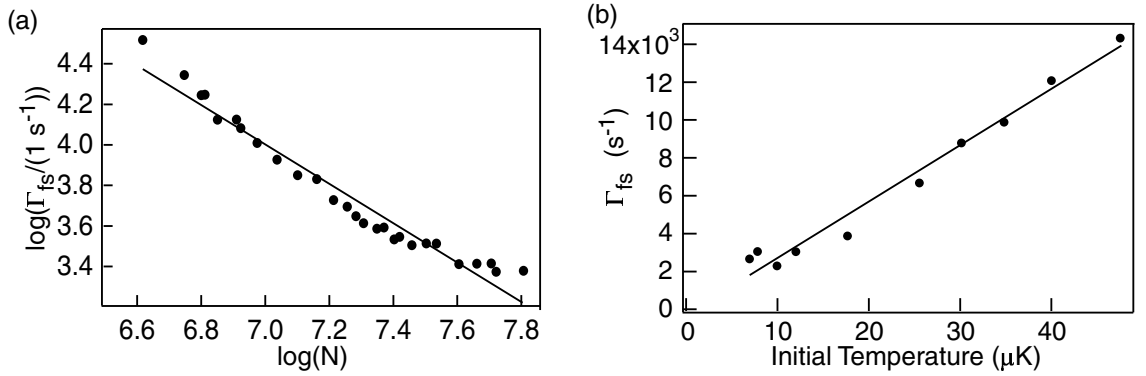

Figure 5: (a) Log-log plot of the threshold free-space scattering rate versus the atom number $N$. The linear fit has slope $-0.97 \pm .04$. Here $T=5.6 \mu \mathrm{K}, \delta_{a} / 2 \pi=-2.4 \mathrm{GHz}$. (b) Threshold free-space scattering rate versus temperature. The parameters are $\delta_{a} / 2 \pi=-2.4 \mathrm{GHz}, N=3.2 \times 10^{7}$.

perature, with all other parameters held fixed (Fig. 5b). The temperature is varied by applying a $200 \mu$ s pulse of near-resonance MOT light with variable intensity. As expected from the recoil-induced gain and superradiance models, the threshold scattering rate is proportional to sample temperature, since the latter determines the maximum amount of inversion between neighboring momentum classes connected by the photon recoil ${ }^{9,18}$.

We have no simple way of verifying the functional dependence on the last remaining parameter, the single-atom scattering probability into the resonator $\eta_{s}{ }^{16}$. This quantity is a function of the solid angle subtended by the simultaneously resonant cavity modes, and the mirror reflectivity, and we cannot easily change the properties of the resonator situated inside the vacuum chamber. However, the measured proportionality coefficient $C_{\text {exp }}=1.6 \pm 0.8$ is close to the value $C_{s r}=2.1$ predicted by the superradiance model, which can serve as an indirect verification of the dependence on cavity properties.

In conclusion, we have observed spatial self-organization of a collection of twolevel atoms inside an optical resonator when irradiated by laser light from the side. This mechanism, elucidated by Domokos and Ritsch ${ }^{10}$, explains our earlier findings 8 of anomalously large collective cavity emission by a non-inverted sample for twolevel atoms. The self-organization leads to strong collective Bragg scattering into the resonator at a rate that far exceeds the single-atom scattering rate for the same parameters. The collective scattering, in turn, is accompanied by a large collective force cooling the sample's center-of-mass motion. The measured variation of the selforganization threshold with atomic and light parameters agrees with that expected from a simple superradiance model. Using these dependencies, we can predict the threshold for other samples, and calculate the necessary laser power to damp the center-of-mass motion of new atomic species.

\section{Acknowledgments}

We would like to thank Helmut Ritsch for interesting discussions. This work was funded in parts by the ARO. 


\section{References}

1. S. Chu, C. Cohen-Tannoudji, W.D. Phillips, Rev. Mod. Phys. 70, 685 (1998).

2. R. Loudon, The Quantum Theory of Light (Oxford University Press, Oxford, 3rd edition, 2000), p. 342f.

3. R. H. Dicke, Phys. Rev. 93, 99 (1954).

4. M. Gangl, P. Horak, and H. Ritsch, J. Mod. Optics 47, 2741 (2000).

5. E. M. Purcell, Phys. Rev. 69, 681 (1946).

6. P. Goy, J. M. Raimond, M. Gross, and S. Haroche, Phys. Rev. Lett. 50, 1903 (1983).

7. D. J. Heinzen, J. J. Childs, J. E. Thomas, and M. S. Feld. Phys. Rev. Lett. 58, 1320 (1987).

8. A. T. Black, H. W. Chan, and V. Vuletić, in Proceedings of the XVIII International Conference on Atomic Physics 2002, eds. H. R. Sadeghpour, E. J. Heller, and D. E. Pritchard (World Scientific, Singapore, 2003), p. 91.

9. J.-Y. Courtois, G. Grynberg, B. Lounis, and P. Verkerk, Phys. Rev. Lett. 72, 3017 (1994).

10. P. Domokos and H. Ritsch, Phys. Rev. Lett. 89, 253003 (2002).

11. A.T. Black, H.W. Chan, and V. Vuletić, submitted to Phys. Rev. Lett. (2003).

12. D. S. Weiss, E. Riis, K. A. Moler, and S. Chu, in Light Induced Kinetic Effects on Atoms, Ions, and Molecules, eds. I. Moi, S. Gozzini, C. Gabbanini, E. Arimondo, and F. Strumia (ETS Editrice, Pisa, 1991), p. 35.

13. H.W. Chan, A.T. Black, and V. Vuletić, in preparation.

14. V. Vuletić and S. Chu, Phys. Rev. Lett. 84, 3787 (2000).

15. P. Horak, G. Hechenblaikner, K. M. Gheri, H. Stecher, and H. Ritsch, Phys. Rev. Lett. 79, 4974 (1997).

16. V. Vuletić, H. W. Chan, and A. T. Black, Phys. Rev. A 64, 033405 (2001).

17. Th. Elsässer, B. Nagorny, and A. Hemmerich, Phys. Rev. A 67, 051401(R) (2003).

18. P. R. Berman, Phys. Rev. A 59, 585 (1999).

19. S. Haroche and J. M. Raimond, Adv. At. Mol. Opt. Phys. 20, 347 (1985). 